\title{
DEVELOPMENT AND CHARACTERIZATION OF NOVEL HERBAL FORMULATION (POLYMERIC MICROSPHERES) OF SYZYGIUM CUMINI SEED EXTRACT
}

\author{
RANU BISWAS ${ }^{*}$, KALYAN KUMAR SEN ${ }^{2}$
}

1Department of Pharmaceutics, BCDA College of Pharmacy and Technology, Kolkata 700127, WB, India, 2Department of Pharmaceutics, Gupta College of Technological Sciences, Asansol 713301, WB, India

Email: ranubiswas2008@gmail.com

Received: 19 Jul 2018, Revised and Accepted: 07 Aug 2018

\begin{abstract}
Objective: The purpose of the present investigation was to develop and characterize a novel herbal formulation (polymeric microspheres) of Syzygium cumini seed extract.

Methods: The extract-loaded microspheres using biological macromolecule ethyl cellulose (EC) was prepared by o/w emulsion solvent evaporation technique using polyvinyl alcohol (PVA) emulsifier. The effect of various process and formulation variables (stirring speed, evaporation time, drug/polymer ratio and organic/aqueous phase ratio) on the properties of microspheres was evaluated.

Results: Micromeritic properties indicated good flow properties, and scanning electron microscopy (SEM) confirmed the spherical nature of the prepared microspheres. The particle size and entrapment efficiency were varied between 34.25 to $176.25 \mu \mathrm{m}$ and 10.51 to $42 \%$ depending upon the variables. All the formulations showed minimal drug release in an acidic environment (pH 1.2) confirming the prevention of drug release in the stomach and enteric nature of the polymer. Sustained drug release has been observed in alkaline dissolution media (pH 7.4) after 12 h of drug release study except for formulation F7 which contains a lower concentration of polymer. The fourier transform infrared spectroscopy (FTIR) analysis indicated the compatibility of the extract with the polymer. The absence of extract-polymer interaction was indicated by the differential scanning calorimetry (DSC) thermogram. x-ray diffraction (XRD) analysis revealed the amorphous nature of the extract in the microspheres which in pure form exhibits a crystalline structure.
\end{abstract}

Conclusion: The findings of this present study suggest that microsphere formulation was a promising carrier for novel delivery of herbal drugs.

Keywords: Polymeric microspheres, Ethylcellulose, Syzygium cumini, Herbal formulation, Solvent evaporation, Polyvinyl alcohol, Phytopharmaceuticals, Novel drug delivery system

(C) 2018 The Authors. Published by Innovare Academic Sciences Pvt Ltd. This is an open access article under the CC BY license (http://creativecommons.org/licenses/by/4.0/) DOI: http://dx.doi.org/10.22159/ijap.2018v10i5.28624

\section{INTRODUCTION}

Herbal medicines are the oldest form of health care and the future of medicine is rooted in the past. Herbs are staging a comeback and herbal "renaissance" is happening all over the globe. People are being interested in herbal therapies rather mainstream medicine due to there is a growing concern over the reliance and safety of drugs and surgery as well as many natural measures produce better results than drugs/surgery without the side effects [1]. Diabetes mellitus (DM), the third "killer" of mankind, is an important chronic metabolic disorder (also called lifestyle disease) affecting carbohydrate, protein and fat metabolism. The worldwide survey reported that the DM is affecting nearly $10 \%$ of the populations. The diabetic people are not able to produce or properly use insulin in the body, so they have a high level of blood glucose. DM is managed by oral hypoglycemic agents which have a characteristic profile of serious side effects [2]. So, despite considerable progress in the management of DM by synthetic drugs, the search for indigenous natural antidiabetic agents is still going on. Ethnobotanical information also indicates that more than 800 plants are used as traditional remedies for the treatment of diabetes throughout the world [3]. Recently herbal medicines have acquired more importance in the treatment of diabetes as they are free from side effects and less expensive when compared to synthetic hypoglycemic agents [4]. Syzygium cumini (L.) seed which is rich in polyphenols and flavonoids, has been reported to be widely used as the traditional system of medicine to treat diabetes [5]. Conventional formulation (powder dosage form) of Syzygium cumini seed is available commercially and the diabetic people widely use it to regulate their blood glucose level. Drugs of natural origin suffer from poor physicochemical properties, which are related to their poor bioavailability, diminishing their therapeutic potency despite their extraordinary potential [6]. Generally to overcome these limitations, developing the novel herbal drug delivery system
(NHDDS) with better absorption profile is of premier importance [1]. These novel formulations are advantageous over conventional formulations regarding enhancement of solubility, stability, membrane permeability, bioavailability, improved pharmacological activity through sustained release profile and reduced toxicity [6] The novel carriers firstly, deliver the drug at a predetermined rate over the period of treatment and secondly, channel the active entity to the site of action. Whereas the conventional dosage forms are unable to achieve these. Micro/nano-sized NHDDS have a potential future for enhancing the activity and overcoming problems associated with plant medicines [1].

In the past, herbal drugs did not attract researcher's interest in the development of NDDS due to difficulties in processing (including standardization, extraction, and identification). However, with the advancement of modern scientific techniques, recently new doors have been opened for the development of novel herbal formulation [7]. Today one of the most attractive areas of research in drug delivery is the design of polymeric microspheres that are able to deliver the drug to the target site, at the right time and right doses [8]. Ethyl cellulose is a semi-synthetic, lipophilic, non-toxic, biocompatible, non-degradable, cost-effective polymer and has been widely used for both lipophilic and hydrophilic drugs in the preparation of controlled release dosage form. Among all other methods used to prepare microspheres, solvent evaporation technique is the simplest one [9].

No attempts have been made for the development of the novel formulation of Syzygium cumini seed extract. In this study, we have tried to develop a smart delivery system for the extract. So, the objective of the present work was to develop and characterize novel herbal formulation (polymeric microspheres) of Syzygium cumini seed extract by emulsion solvent evaporation technique using ethyl cellulose as the polymer. 


\section{MATERIALS AND METHODS}

\section{Materials}

Syzygium cumini seed powder (Vyas Pharmaceuticals, a GMP certified unit, Indore, MP, India), Ethylcellulose (Loba Chemie Pvt. Ltd.), Polyvinyl alcohol (Nice Chemicals P Ltd.), Dichloromethane (Loba Chemie Pvt. Ltd.). All other chemicals used were of analytical grade.

\section{Preparation of extract}

The Syzygium cumini seed powder was extracted with ethanol: water (70:30) at room temperature by maceration for $14 \mathrm{~d}$. The extract was then filtered and concentrated by a rotary vacuum evaporator at a temperature of 400C. It was then kept in the refrigerator in an airtight container for further studies.

\section{Preparation of extract-loaded ethyl cellulose microspheres}

Extract-loaded polymeric microspheres were prepared at room temperature by most widely used o/w emulsion solvent evaporation technique $[10,11]$. Different formulations were prepared by dissolving the polymer and drug in dichloromethane (DCM) as specified in table 1 . This polymeric drug solution (internal oil phase) was emulsified by pouring slowly into external aqueous phase containing $1 \% \mathrm{w} / \mathrm{v}$ polyvinyl alcohol (PVA) as an emulsifying agent, with continuous stirring using propeller type electric stirrer (Remi, Mumbai, India). The stirring speed and evaporation time were specified in table 1. During the emulsification process, the dispersed phase was converted into minute droplets and then solidified into rigid microspheres due to evaporation of the organic solvent. The resulting microspheres were recovered by filtration, washed three times with double distilled water to remove any residual PVA and dried at room temperature. All the formulations were prepared in triplicate and further characterized.

\section{Characterization of microspheres}

\section{Production yield}

After drying the obtained microspheres were weighed accurately. The $\%$ yield was determined by using the following formula:

$$
\% \text { yield }=\frac{\text { Massofmicrospheresobtained }}{\text { Totalmassofdrug }+ \text { polymer }} * 100
$$

Table 1: Formulation design of extract-loaded ethyl cellulose microspheres

\begin{tabular}{|c|c|c|c|c|}
\hline Batch code & Stirring speed (rpm) & Evaporation time (h) & Drug/polymer ratio & Organic/aqueous phase ratio \\
\hline F1 & 800 & 3 & $1: 2$ & $1: 3$ \\
\hline $\mathrm{F} 2^{\mathrm{a}}$ & 1000 & 3 & $1: 2$ & $1: 3$ \\
\hline F3 & 1500 & 3 & $1: 2$ & $1: 3$ \\
\hline $\mathrm{F} 4$ & 1000 & 1 & $1: 2$ & $1: 3$ \\
\hline F5 & 1000 & 2 & $1: 2$ & $1: 3$ \\
\hline$F 6^{a}$ & 1000 & 3 & $1: 2$ & $1: 3$ \\
\hline F7 & 1000 & 3 & $1: 1$ & $1: 3$ \\
\hline F8 & 1000 & 3 & $1: 2$ & $1: 3$ \\
\hline F9 & 1000 & 3 & $1: 3$ & $1: 3$ \\
\hline $\mathrm{F} 10^{\mathrm{a}}$ & 1000 & 3 & $1: 2$ & $1: 3$ \\
\hline F11 & 1000 & 3 & $1: 2$ & $1: 5$ \\
\hline F12 & 1000 & 3 & $1: 2$ & $1: 10$ \\
\hline
\end{tabular}

aSame formulation variables.

\section{Drug entrapment efficiency (DEE)}

Entrapment efficiency was determined by dissolving $10 \mathrm{mg}$ of microspheres in $2 \mathrm{ml}$ DCM. When the particles were completely dissolved, the mixture was diluted up to $50 \mathrm{ml}$ with phosphate buffer $\mathrm{pH}$ 7.4 and stirred for $1 \mathrm{~h}$ by using a magnetic stirrer for complete removal of DCM. The polymer was removed by filtration. The absorbance of the filtrate was measured at $760 \mathrm{~nm}$, and the content was determined by using a calibration curve of gallic acid, which has been considered as a marker peak for Jamun and its formulations.

$$
\% \text { Drugentrapment }=\frac{\text { Calculateddrugcontent }}{\text { Theoreticaldrugcontent }} * 100
$$

\section{Micromeritic properties}

\section{Bulk density}

A measured quantity of microspheres was introduced into $10 \mathrm{ml}$ measuring cylinder, and the volume was noted. After that, it was tapped manually 100 times. Again the volume was noted. The loose and tapped bulk density was determined by using the following formulas:

$$
\begin{aligned}
& \text { Loosebulkdensity }(L B D)=\frac{\text { Weightofmicrospheres }}{\text { Bulkvolume }} \\
& \text { Tappedbulkdensity }(T B D)=\frac{\text { Weightofmicrospheres }}{\text { Tappedvolume }}
\end{aligned}
$$

\section{Carr's index}

Indirect measurement of rheological properties like shape, size, surface area, moisture content and cohesiveness of materials is done by using Carr's index. It is also known as Carr's compressibility index (Ci).

$$
\text { Carr'sin dex }=\frac{\text { Tappeddensity }- \text { bulkdensity }}{\text { Tappeddensity }} * 100
$$

It reflects the flow characteristics of materials. $\mathrm{C}_{\mathrm{i}}<15 \%$-good flow

$\mathrm{C}_{\mathrm{i}>} 25 \%$-poor flow.

\section{Hausner's ratio}

Another index for measuring flowability of materials.

$$
\text { Hausner' } \operatorname{sratio}(H)=\frac{\text { Tappeddensity }}{\text { Bulkdensity }}
$$

$\mathrm{H}>1.25$-poor flow.

\section{Angle of repose}

The maximum possible angle between the surface of the pile and the horizontal plane is known as the angle of repose. It was determined by the fixed funnel method using the following formula:

$$
\tan \theta=\frac{h}{r}
$$

Where,

$$
\begin{aligned}
& \mathrm{h}=\text { height of the pile } \\
& \mathrm{r}=\text { radius of the pile }
\end{aligned}
$$

\section{Particle size analysis}

The particle size of the prepared microspheres was measured by the optical microscope with the aid of an eye-piece micrometer which was previously calibrated with the stage micrometer. The mean particle size and size distribution were determined. 


\section{In vitro drug release studies}

The drug release profile of formulated microspheres was carried out by using USP dissolution test apparatus type II rotating paddle. The test was performed both in $0.1 \mathrm{~N} \mathrm{HCl}(\mathrm{pH} 1.2)$, and phosphate buffer $(\mathrm{pH} 7.4)$ as dissolution media $(900 \mathrm{ml})$ maintained at $37 \pm 0.5{ }^{\circ} \mathrm{C}$ and $100 \mathrm{rpm}$. The samples were withdrawn at predetermined time intervals, filtered and analyzed spectrophotometrically at $760 \mathrm{~nm}$. Cumulative \% drug release (CPR) was calculated from the standard curve.

\section{Drug-excipients interaction study by FTIR}

The FTIR spectra were taken to investigate the possible interaction between drug and polymer in the microspheres formulation. The spectra of the extract, polymer, empty microspheres and extractloaded microspheres were recorded in the range of $4000-400 \mathrm{~cm}^{-1}$ from $\mathrm{KBr}$ pellet using FTIR (Bruker Alpha T).

\section{Surface morphology of microspheres (SEM)}

Scanning electron microscopy is an excellent tool to examine the shape and surface morphology of the microspheres. Prior to observation, the sample was placed on an adhesive stub. The SEM photograph was taken at a working distance of $15 \mathrm{~mm}$; the acceleration voltage is $15 \mathrm{kV}$ with secondary electron image (SEI) detector.

\section{X-ray diffraction analysis (XRD)}

The X-RD pattern of the extract and the microspheres were obtained by X-ray diffractometer. The samples were irradiated with monochromatized X-ray $\left(\mathrm{Cu} \mathrm{K}_{\infty}\right)$ with scanning rate of $2^{\circ} \mathrm{min}^{-1} \mathrm{in}$ the range of $5-70{ }^{\circ} \mathrm{C}(2 \theta$ diffraction angle). The voltage was $40 \mathrm{kV}$ and current $30 \mathrm{~mA}$.

\section{Differential scanning calorimetry (DSC)}

Thermal analysis of pure extract and extract-loaded formulation was performed with differential scanning calorimeter to determine the thermal properties of the drug and that of the formulation. The sample was placed into the aluminum container and heated in the range of $20-300{ }^{\circ} \mathrm{C}$ at a constant rate of $10{ }^{\circ} \mathrm{C} / \mathrm{min}$ under nitrogen atmosphere.

\section{Drug release kinetics studies}

Drug release mechanism from the microspheres was studied by fitting the in vitro release data of optimized formulation to different mathematical models such as Zero order (\% cumulative drug release vs. time), first order (log \% drug retained vs. time), Higuchi model (\% cumulative drug release vs. square root of time) and Peppas exponential equation (log \% drug release vs. log time). All curve fitting, calculation, and plotting were performed using Microsoft excel solver, and regression coefficient $\left(\mathrm{R}^{2}\right)$ values were calculated.

\section{RESULTS AND DISCUSSION}

\section{Formulation of microspheres}

The solvent evaporation method, most common and patented, was selected for the preparation of ethyl cellulose microspheres for novel delivery of Syzygium cumini seed extract. Though the method is simple, many factors have been shown to influence the properties of the prepared microspheres [12]. Size is one of the most important factors that should be considered in the design and development of this system as size affects the interaction with the biological system, entry of the drug and drug release. Another important parameter is the entrapment efficiency (EE) which in turn related to the productivity of the pharmaceutical system [13]. The extract loaded ethyl cellulose microspheres were prepared successfully by $\mathrm{o} / \mathrm{w}$ emulsion solvent evaporation method. Microspheres were formed by pouring a polymeric drug solution in dichloromethane, into the aqueous solution of PVA. The evaporation of entrapped dichloromethane leads to the formation of microspheres [14]. This formed smooth surfaced, spherical shaped microspheres. All the batches were prepared according to the formulation design (table 1). The desirable characteristics of the prepared microspheres are high EE, small particle size, and controlled drug release. As various formulation and processing variables influenced the properties of microspheres, the present work focused towards the systematic investigation of the parameters which could influence the microspheres characteristics. Gallic acid was used as a marker peak for Jamun and its formulations [15].

\section{Production Yield}

The percentage yield for all the formulations was found to be in the range of $39.53-58.79 \%$ (table 2), and it was increasing with increased polymer concentration and evaporation time. It decreased when the external aqueous phase volume increased. When the stirring rate was increased from 800 to $1000 \mathrm{rpm}, \%$ yield increased (52.23-54.75\%), but on further increase in the rate to $1500 \mathrm{rpm}$, it decreased $[16,17]$.

\section{Micromeritic properties}

The micromeritic properties for all the formulations were expressed in terms of bulk density, tapped density, Carr's index, Hausner's ratio and angle of repose. As given in table 2, the values of Carr's index were found to be in the range of $11.3-15.66 \%$, indicating good compressibility. Hausner's ratio was recorded below 1.25, which represents good flowability. The angle of repose was found to be below $30^{\circ}$, showing the free-flowing nature of the microspheres. The micromeritic properties of all the formulations indicated that microspheres were free flowing in nature. The similar finding was also reported previously $[9,16]$.

Table 2: Production yield and flow properties of extract-loaded ethyl cellulose microspheres

\begin{tabular}{|c|c|c|c|c|c|c|}
\hline Batch code & $\%$ yield & $\begin{array}{l}\text { Bulk density } \\
(\mathrm{g} / \mathrm{ml})\end{array}$ & $\begin{array}{l}\text { Tapped density } \\
(\mathrm{g} / \mathrm{ml})\end{array}$ & Carr's index (\%) & Hausner's ratio & Angle of repose $\left({ }^{\circ}\right)$ \\
\hline F1 & 52.23 & $0.455 \pm 0.42$ & $0.512 \pm 0.13$ & 11.13 & 1.12 & $18.53 \pm 0.62$ \\
\hline F2 & 54.75 & $0.153 \pm 0.59$ & $0.175 \pm 0.15$ & 12.57 & 1.14 & $20.12 \pm 0.73$ \\
\hline F3 & 45.23 & $0.116 \pm 0.32$ & $0.136 \pm 0.21$ & 14.71 & 1.17 & $22.57 \pm 0.55$ \\
\hline $\mathrm{F} 4$ & 42.35 & $0.149 \pm 0.15$ & $0.168 \pm 0.35$ & 11.31 & 1.13 & $21.44 \pm 0.63$ \\
\hline F5 & 49.69 & $0.134 \pm 0.64$ & $0.158 \pm 0.51$ & 15.18 & 1.18 & $23.97 \pm 0.81$ \\
\hline F6 & 54.75 & $0.153 \pm 0.29$ & $0.175 \pm 0.34$ & 12.57 & 1.14 & $20.12 \pm 0.93$ \\
\hline F7 & 40.31 & $0.147 \pm 0.31$ & $0.171 \pm 0.43$ & 14.04 & 1.16 & $23.56 \pm 0.57$ \\
\hline F8 & 54.75 & $0.153 \pm 0.42$ & $0.175 \pm 0.33$ & 12.57 & 1.14 & $20.12 \pm 0.75$ \\
\hline F9 & 58.79 & $0.218 \pm 0.23$ & $0.256 \pm 0.36$ & 14.84 & 1.17 & $24.31 \pm 0.79$ \\
\hline F10 & 54.75 & $0.153 \pm 0.51$ & $0.175 \pm 0.25$ & 12.57 & 1.14 & $20.12 \pm 0.61$ \\
\hline F11 & 42.21 & $0.169 \pm 0.23$ & $0.195 \pm 0.19$ & 13.33 & 1.15 & $24.92 \pm 0.57$ \\
\hline F12 & 39.53 & $0.183 \pm 0.29$ & $0.217 \pm 0.35$ & 15.66 & 1.19 & $25.14 \pm 0.73$ \\
\hline
\end{tabular}

Data represent mean \pm standard deviation (SD), $\mathrm{n}=3$.

\section{Drug entrapment efficiency (DEE)}

All the formulations were evaluated for entrapment efficiency (EE), and the results are illustrated in table 3 , which shows that the polymer concentration and evaporation time are directly proportional to the EE, whereas the stirring speed and organic/aqueous phase volume ratio are inversely proportional to the EE [18]. High polymer concentration increases the viscosity of the organic phase, which increases the diffusional resistance to the drug molecules, thereby reducing the partitioning of the drug into 
external aqueous phase and entrapping more drug in polymeric microspheres [12]. In another way, with the increase in polymer concentration, the particle size increases. This causes the decrease in the surface area to be exposed to water. Thus, the drug loss due to diffusion is reduced and enhanced entrapment [13]. As the drug: polymer ratio increased from $1: 1$ to $1: 2$ and $1: 3$. The mean EE increased from $18 \%$ to $30.03 \%$ and $42 \%$ respectively.

Evaporation of organic solvent for the shorter period of time causes rapid replacement of the organic solvent with the aqueous medium before the droplet hardening occurs. This results in reduced drug entrapment. The lowest entrapment (15.01\%) was observed when the evaporation time was $1 \mathrm{~h}$. Upon increasing the aqueous phase volume, more amount of drug is dissolved in the aqueous medium. Therefore, increasing the drug loss from organic phase results in the reduction of the EE [19]. Increased stirring speed delivers greater energy to the system, resulting in the faster breakdown of the formed microparticles and lowers the drug entrapment [20].

\section{Particle size}

The particle size was determined by optical microscopy using eyepiece micrometer which was previously calibrated with the stage micrometer. The particle size was measured in the range of 34.25$176.25 \mu \mathrm{m}$ among different formulations. The mean particle size of the microspheres significantly increased ( 34.25 to $114.75 \mu \mathrm{m}$ ) with an increase in polymer concentration. Higher polymer concentration with the fixed volume of organic solvent causes more viscous dispersion, leading to the formation of larger emulsion droplet and consequently larger particles. The viscous forces oppose the shear stresses in the organic phase, and the final particle size depends on the net shear stress available for droplet breakdown [21]. A critical parameter determining the particle size is diffusion of the organic solvent through the interface of emulsion droplet. For shorter evaporation time, the diffusion may not have been completed before the droplets start to harden, thus forming larger particles. But when the organic solvent is allowed to evaporate for a long time, the extent of diffusion will be greater, resulting in a smaller particle size. An increase in the particle size was observed with increased organic/aqueous phase ratio which may be explained by the change in viscosity of the emulsion formed during the process. Increased viscosity resulted in high viscous resistance against the sheer force during the microparticle formation. The external energy (in the form of stirring speed and amount of emulsifier) for droplet breakdown was kept constant. Thus, on increasing the aqueous phase volume, the same amount of energy must be distributed in large volume, causing less droplet breakdown and resulting larger particles. When the stirring rate was increased from 800 to $1500 \mathrm{rpm}$, the particle size was reduced rapidly $(176.25-38.5 \mu \mathrm{m})$. This is because higher energy is released in the process that leads to the rapid breakdown of the emulsion droplets, resulting in smaller particle size [19]. The particle size distribution of the optimized formulation was shown in fig. 2.

Table 3: Comparison of entrapment efficiency, particle size and the cumulative amount of drug release from extract-loaded ethyl cellulose microspheres prepared using various process and formulation variables

\begin{tabular}{llll}
\hline Batch code & Entrapment efficiency(\% $\mathbf{S D})$ & Mean particle size $(\boldsymbol{\mu m} \pm \mathbf{S D})$ & Cumulative drug release at 12h $(\% \mathbf{\% D})$ \\
\hline F1 & $39.03 \pm 2.6$ & $176.25 \pm 58.4$ & $62.30 \pm 1.2$ \\
F2 & $30.03 \pm 2.1$ & $45.5 \pm 26.4$ & $88.30 \pm 0.8$ \\
F3 & $19.52 \pm 1.9$ & $38.5 \pm 20.8$ & $92.50 \pm 1.6$ \\
F4 & $15.01 \pm 3.6$ & $128.95 \pm 43.1$ & $61.20 \pm 2.1$ \\
F5 & $25.52 \pm 2.9$ & $87.5 \pm 35.1$ & $72.47 \pm 1.1$ \\
F6 & $30.03 \pm 2.1$ & $45.5 \pm 26.4$ & $88.30 \pm 0.8$ \\
F7 & $18.00 \pm 4.3$ & $34.5 \pm 23.7$ & $99.22 \pm 1.8^{*}$ \\
F8 & $30.03 \pm 2.1$ & $45.5 \pm 26.4$ & $88.30 \pm 0.8$ \\
F9 & $42.00 \pm 1.9$ & $114.75 \pm 45.9$ & $56.19 \pm 2.0$ \\
F10 & $30.03 \pm 2.1$ & $45.5 \pm 26.4$ & $88.30 \pm 0.8$ \\
F11 & $24.02 \pm 3.2$ & $73.25 \pm 41.6$ & $73.00 \pm 1.7$ \\
F12 & $10.51 \pm 3.9$ & $113.25 \pm 43.4$ & $68.57 \pm 2.1$ \\
\hline
\end{tabular}

Data represent mean \pm standard deviation (SD), $\mathrm{n}=3$. For particle size $\mathrm{n}=100 .{ }^{*} \mathrm{CDR}$ at $8 \mathrm{~h}$.
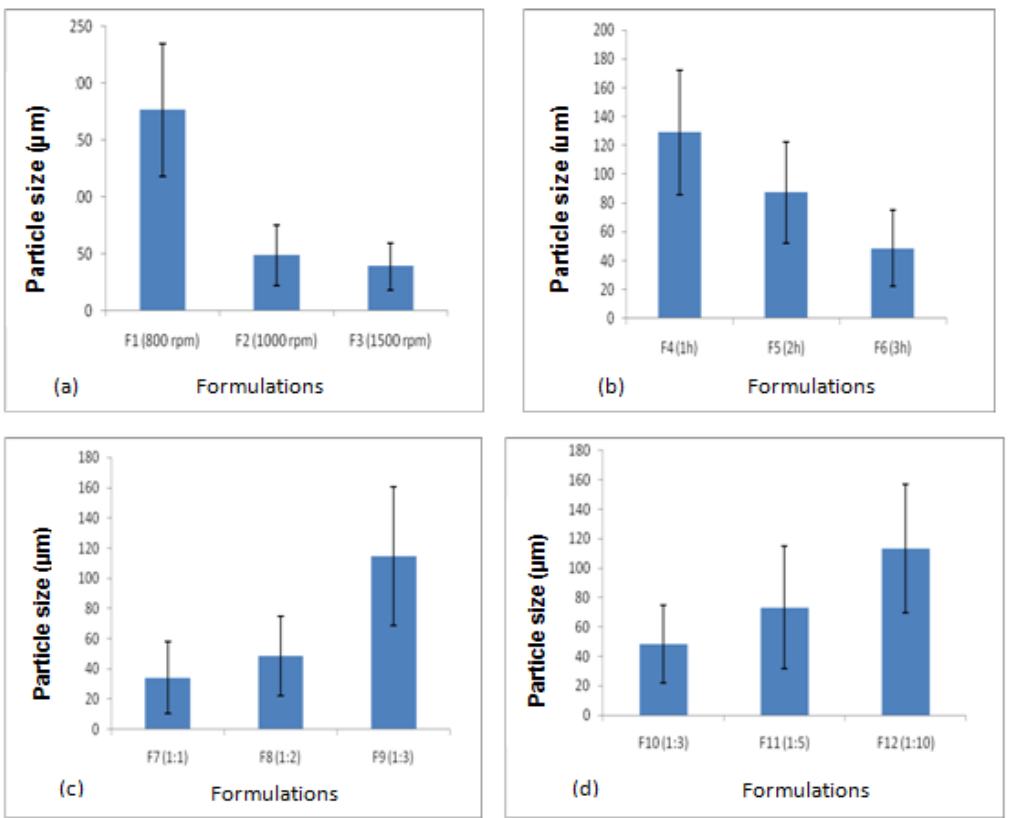

Fig. 1: Effect of (a) Stirring speed, (b) Evaporation time, (c) Drug/polymer ratio and (d) Aqueous/organic phase ratio on the particle size of the extract-loaded ethyl cellulose microspheres (data represent mean $\pm S D$, where $n=3$ ). $S D=$ standard deviation 


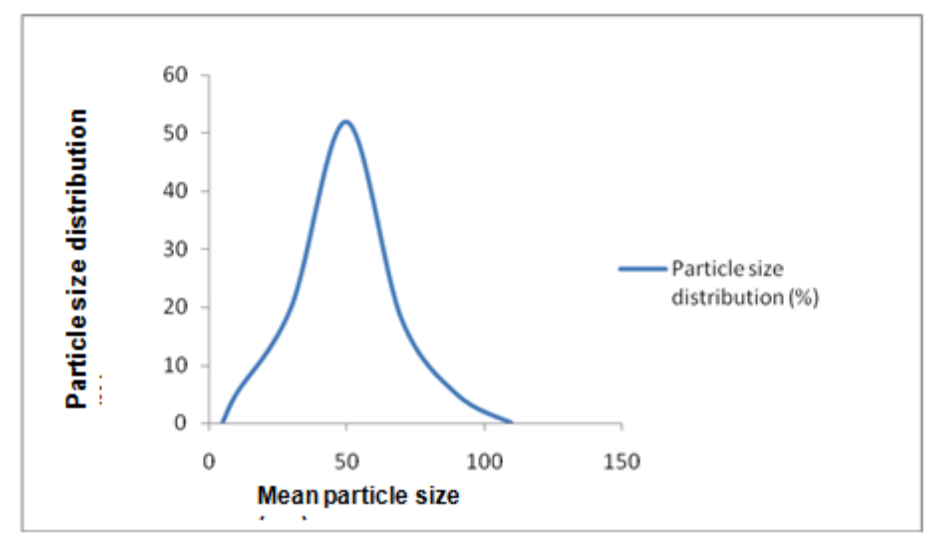

Fig. 2: Particle size distribution of the optimized formulation

\section{In vitro drug release study}

In vitro drug release from extract-loaded ethyl cellulose microspheres was investigated both in gastric $(\mathrm{pH} 1.2)$ and intestinal $(\mathrm{pH}$ 7.4) $\mathrm{pH}$. Dissolution studies were performed for $2 \mathrm{~h}$ at $\mathrm{pH} 1.2$ and $12 \mathrm{~h}$ at $\mathrm{pH} 7.4$. At $\mathrm{pH} 1.2$ negligible amount of drug was released from all the formulations $(<8 \%)$. Whereas at $\mathrm{pH} 7.4$ the maximum drug release $(56.19-99.22 \%)$ indicating the sustained release pattern of the drug from microspheres. The cumulative amount of drug released (CDR) at $12 \mathrm{~h}$ from formulation F7-F9 with drug-polymer ratio $1: 1,1: 2$ and $1: 3$ were $99.22 \%, 88.3 \%$, and $56.19 \%$ respectively. The decreased \% CDR could be due to the increased particle size, resulting in the smaller surface area at high polymer concentration. Another explanation could be the high polymer concentration hinders the drug release by diffusion. Evaporation time influences the particle size and hence affects the drug release. Greater drug release was observed from the formulation F6, prepared with longer evaporation time (3h), due to smaller particle size. Rapid solvent evaporation leads to the smoother surface of the particles as compared to the particles obtained by delayed solvent evaporation. Thus the smoother surface of the particles resulted in the much slower release. Smaller particles were obtained at a high, stirring rate as high speed causes the formation of the finer dispersion of the microparticles. It was explained previously that smaller the particle size, greater the surface area, resulting in faster drug release. Maximum release $(92.5 \%)$ was observed at the speed of $1500 \mathrm{rpm}$, but at $800 \mathrm{rpm}$ that was $62.30 \%$ due to the formation of larger particles. Larger the particles, slower the drug release because of longer diffusion pathways that the drug had to travel to reach dissolution medium. The CDR at $12 \mathrm{~h}$ decreased with increasing aqueous phase volume used in the preparation of microspheres. The difference in release profiles can be attributed to the difference in the surface of the microparticles regardless of the difference in their size. The large volume of aqueous phase causes faster precipitation of the polymer, resulting in the formation of smoother and less porous surface of microparticles. Porosity increases with decreasing aqueous phase volume and thus lower organic/aqueous phase ratio resulted in a more porous surface leading to faster drug release $[13,19]$. The CDR at $12 \mathrm{~h}$ from F10 to F12 prepared with organic/aqueous phase ratio $1: 3,1: 5$ and $1: 10$ were $88.3 \%, 73 \%$, and $68.57 \%$ respectively.
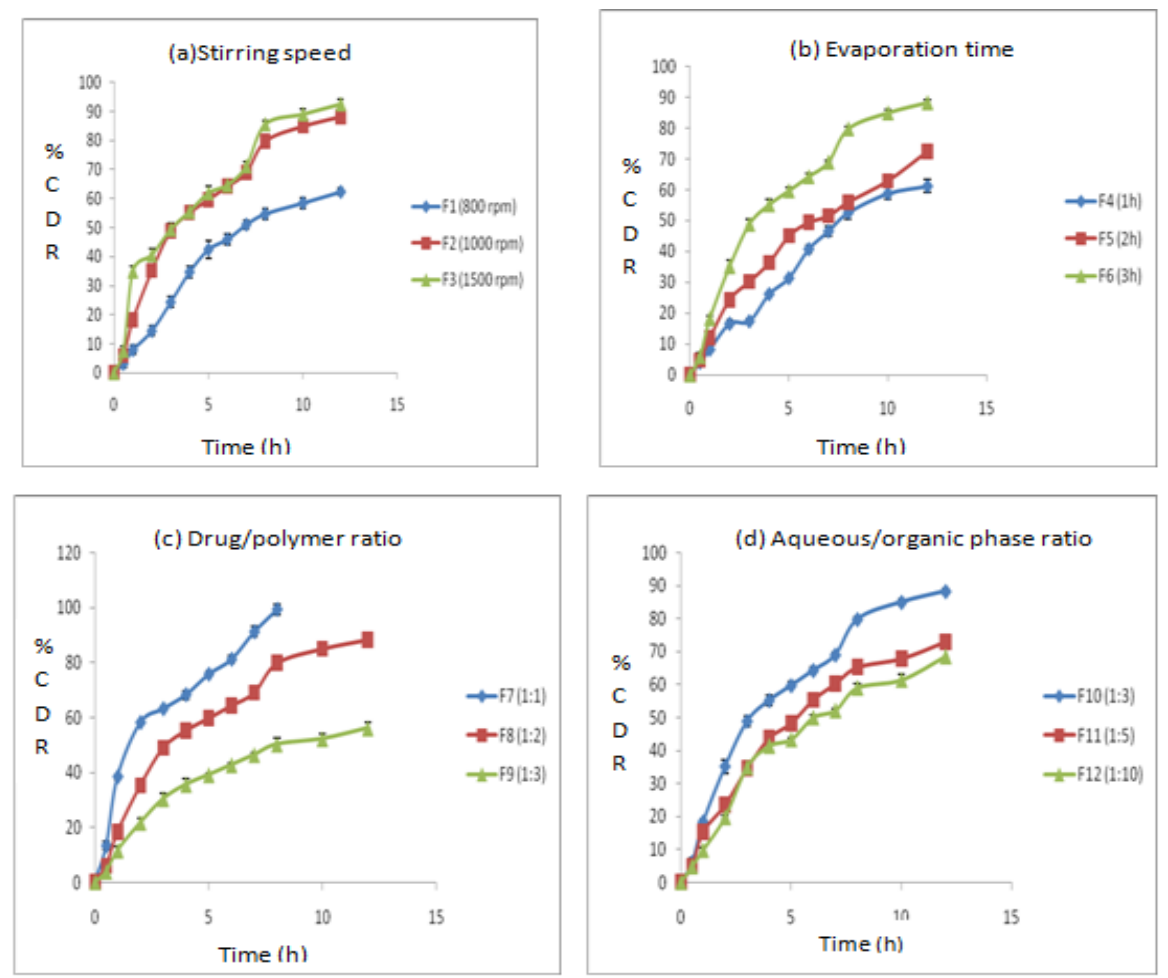

Fig. 3: Effect of (a) Stirring speed, (b) Evaporation time, (c) Drug/polymer ratio and (d) Aqueous/organic phase ratio on in vitro drug release of the extract-loaded ethyl cellulose microspheres (data represent mean $\pm S D$, where $n=3$ ). $S D=s t a n d a r d$ deviation 


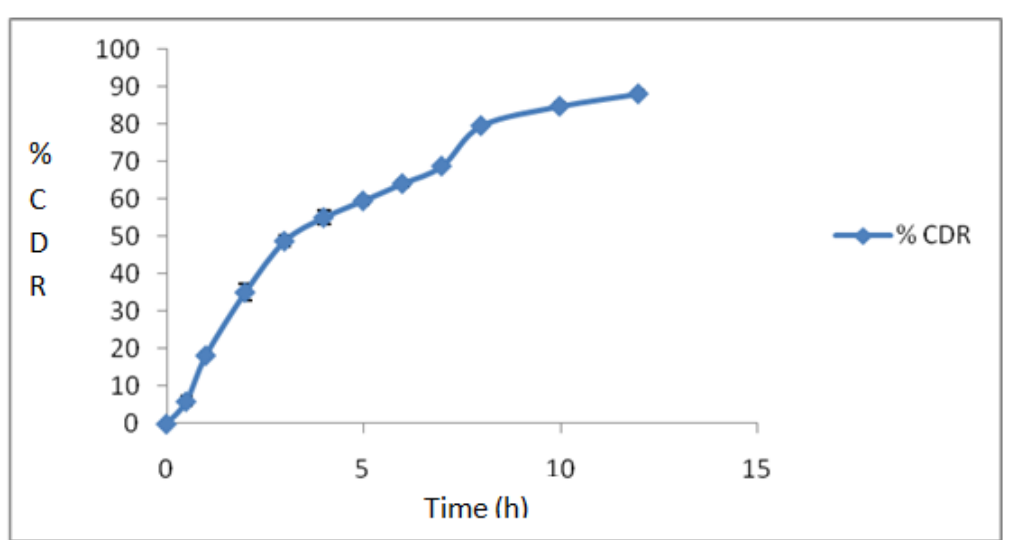

Fig. 4: In vitro drug release profile of optimized formulation (data represent mean $\pm S D$, where $n=3$ ). $S D=$ standard deviation

\section{Release kinetics study}

The dissolution data of the optimized formulation was analyzed according to various model dependent approaches (zero order, first order, Higuchi, and Korsmeyer-Peppas) and the mode of drug release from microspheres was calculated by plotting the curves. The kinetic model with the highest value of the coefficient of determination (R2) was considered to be a more suitable model for dissolution profile. In vitro release data follows first-order kinetics ( $R 2=0.988)$ followed by Higuchi model ( $R 2=0.978)$. Release mechanism was studied by Peppas equation. The value of slope (n) was calculated and found to be greater than 0.89 which indicates the mode of drug release is super case II type in which the erosion of the polymer takes place to release the drug content from the matrix. The first order plot of optimized formulation was shown in fig. 5.

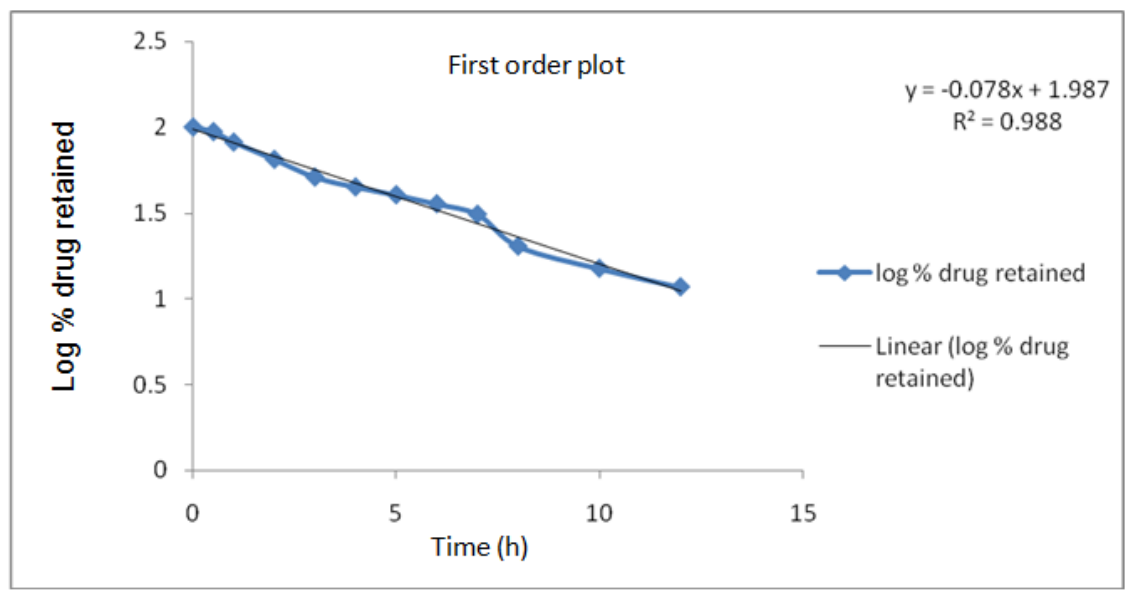

Fig. 5: First order plot of optimized formulation

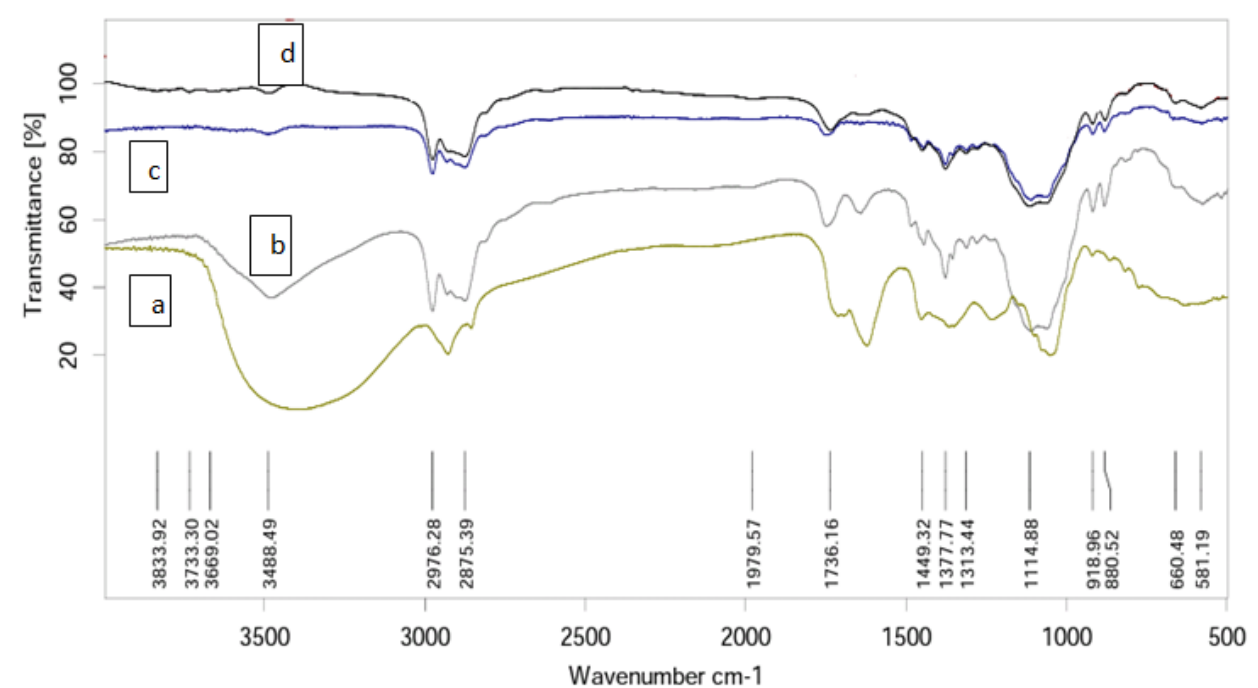

Fig. 6: FTIR spectra of (a) Extract, (b) Polymer (EC), (c) Empty microspheres and (d) Extract-loaded microspheres 


\section{Compatibility study by FTIR spectroscopy}

The compatibility of the extract with the excipients in this formulation was evaluated qualitatively through FTIR analysis. The FTIR spectra obtained for pure extract, polymer, empty microspheres and extract-loaded microspheres, could confirm the chemical compatibility of extract in the ethyl cellulose microspheres. The FTIR spectrum of Syzygium cumini seed extract exhibited absorption in the range of $3404.66 \mathrm{~cm}-1$ to $637.11 \mathrm{~cm}-1$ (fig. 6). The spectrum showed a broad brand around $3404.66 \mathrm{~cm}-1$ assigned to alcohol and hydroxyl group $(\mathrm{OH})$ stretching, which also indicated the presence of phenol and flavonoid. The sharp peak observed at $2929.14 \mathrm{~cm}-1$ indicates the presence of alkane group (C-H). Another sharp peak at $1623.08 \mathrm{~cm}-1$ associated with an $\mathrm{N}-\mathrm{H}$ stretching (primary amines). The peaks at 1451.87 and $1363.00 \mathrm{~cm}-1$
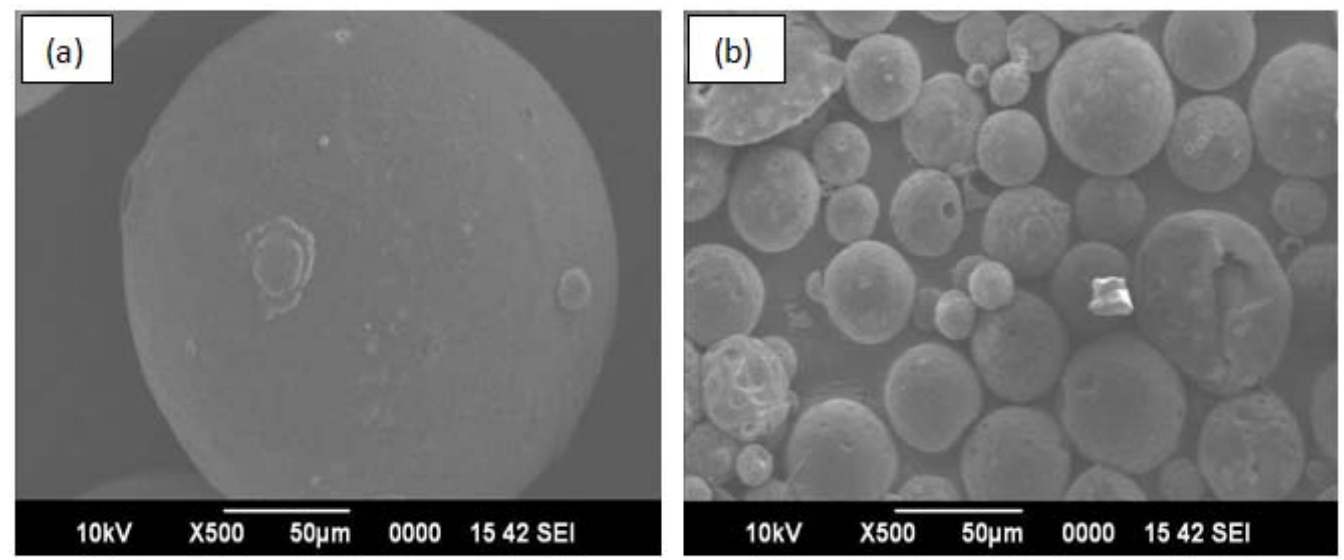

Fig. 7: SEM photographs of EC microspheres: (a) F1, (b) F2

\section{DSC analysis}

DSC studies were performed to understand the nature and interaction of the encapsulated drug in the matrix. The physical state of the drug in the polymer matrix would also influence its release characteristics

[12]. DSC analysis was performed on extract, extract-loaded represent $\mathrm{CH}$ stretching. The sharp peak observed at $1047.98 \mathrm{~cm}-1$ indicates the presence of aliphatic amines (C-N). The peaks observed at 921.01, 773.66 and $637.11 \mathrm{~cm}-1$ represents $\mathrm{C}-\mathrm{H}$ stretching [5]. The characteristic peaks of the extract indicate no major shift of the peak positions, matching the extract-loaded formulation spectrum. Thus the spectral analysis showed that the extract is stable in the ethyl cellulose microspheres.

\section{Surface morphology}

The SEM photograph indicates that the prepared ethyl cellulose microspheres were spherical in shape with a smooth and dense outer surface (fig. 7) without any aggregation. Distinct pores were evident on the surface of microspheres, which will be responsible for drug release. microspheres and the respective thermograms are shown in fig. The extract showed a characteristic peak at $259^{\circ} \mathrm{C}$, which represents the melting point of Gallic acid, but the same was not found in the thermogram of the formulation. A similar observation has been reported by [8]. These results suggested that the extract was dispersed throughout the polymer forming a high energy amorphous state.

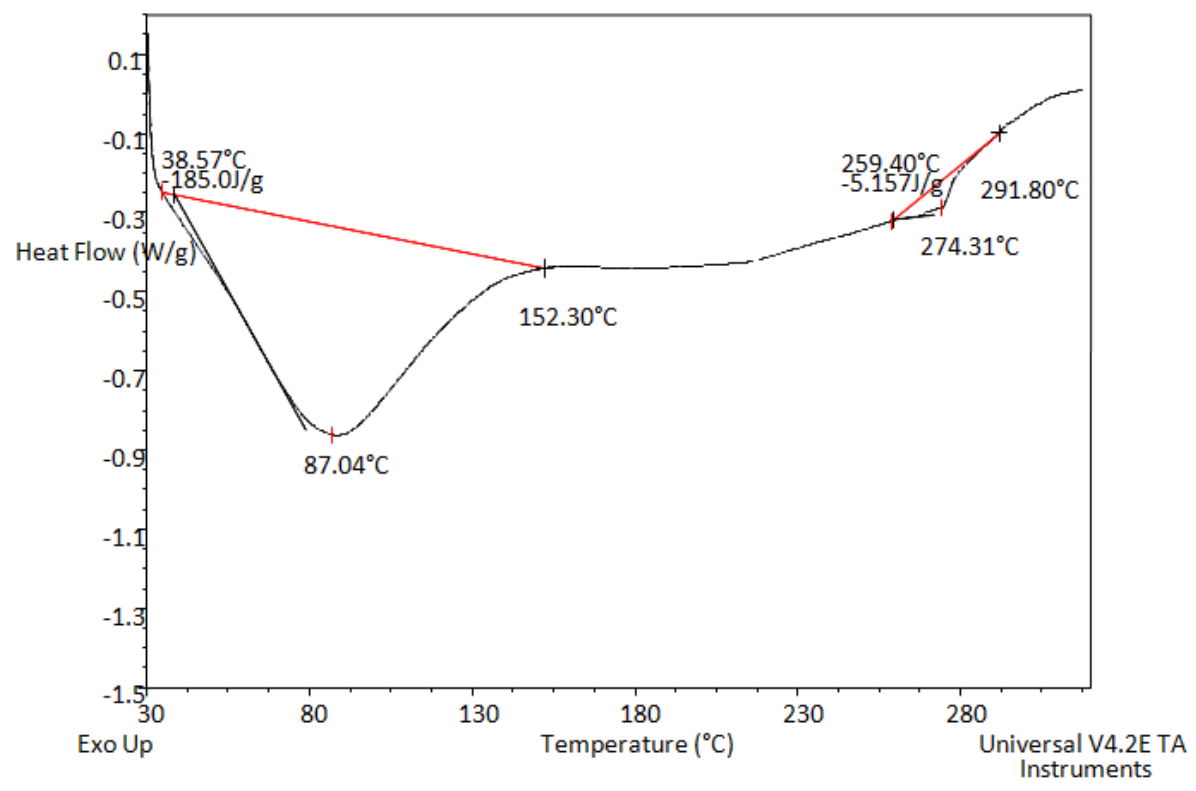

Fig. 8: DSC thermogram of the extract 


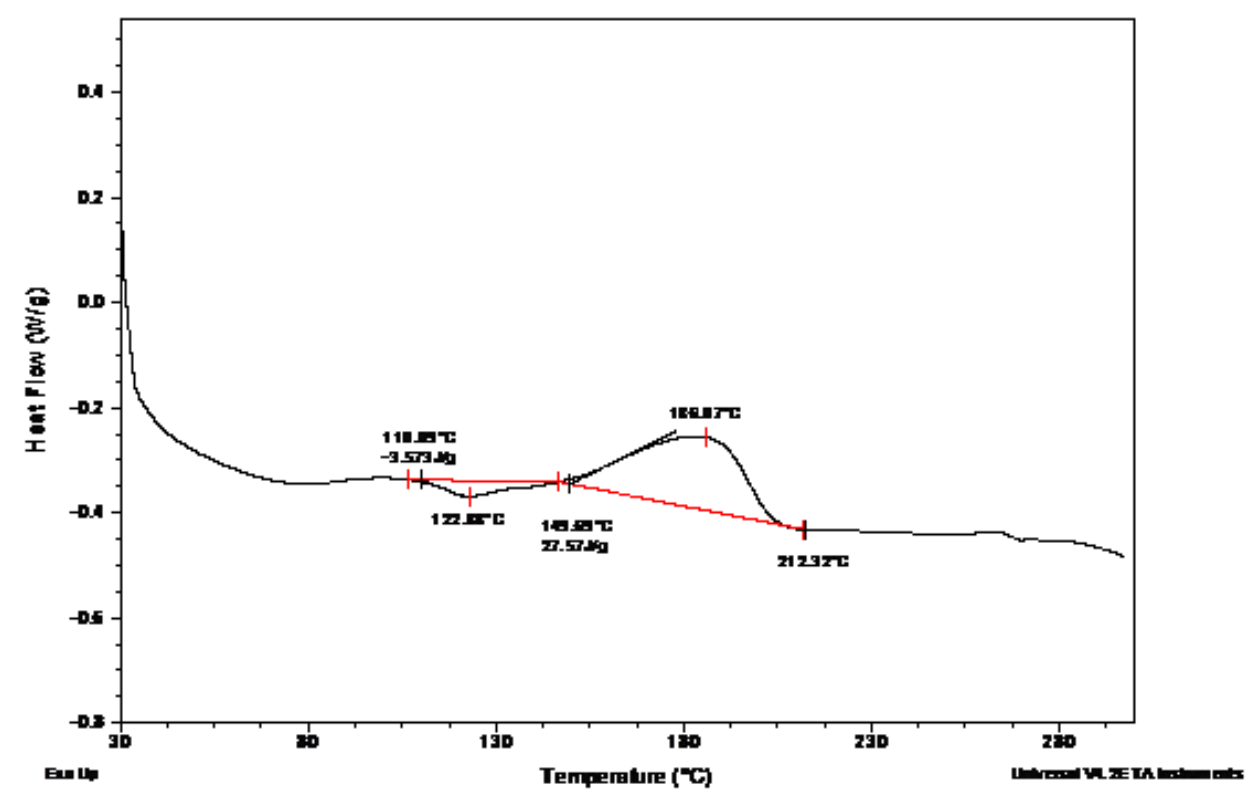

Fig. 9: DSC thermogram of the microspheres

\section{XRD analysis}

X-RD of extract and extract-loaded microspheres were carried out by using X-Ray Diffractometer to find out any change in the crystallinity of drug during microencapsulation. The x-ray diffractogram of extract showed sharp peaks, whereas the formulation decreased the sharpness of peak which indicated that the polymer dispersed the extract at molecular level blended ethyl cellulose microspheres by decreasing the crystallinity of the extract [9].

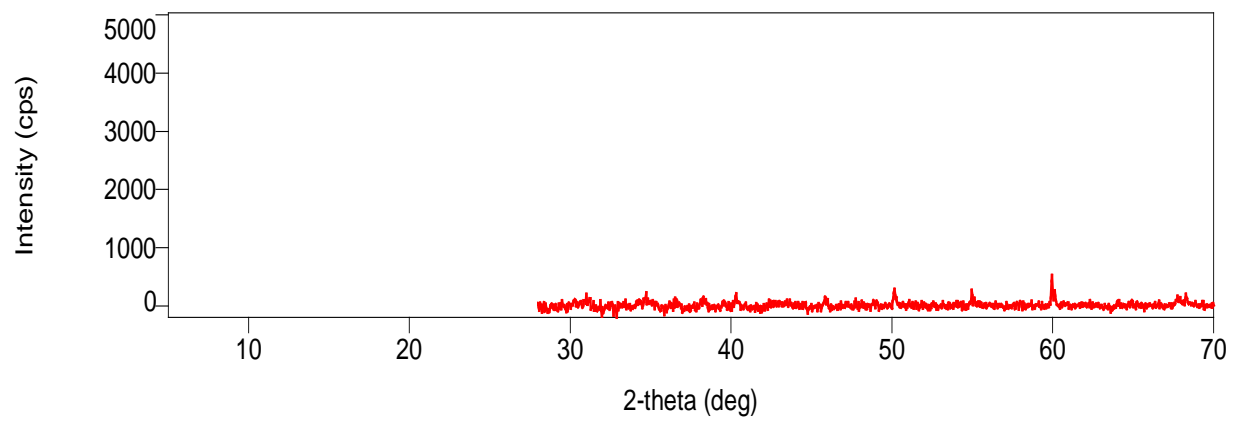

Fig. 10: X-ray diffraction spectra of the extract

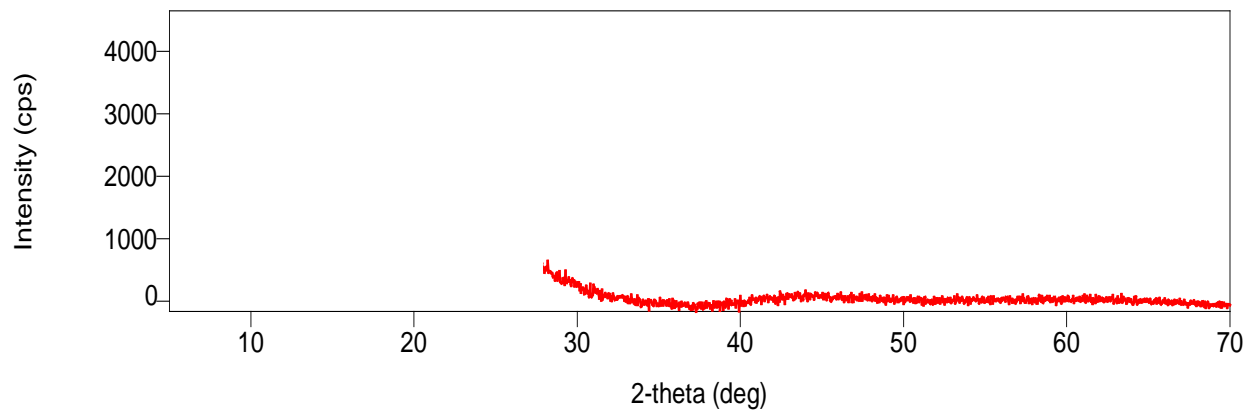

Fig. 11: X-ray diffraction spectra of the microspheres

\section{CONCLUSION}

Extensive work on the formulation and characterization of extractloaded EC microspheres was evaluated. The development of formulation and optimization yields the desired microspheres with sustained drug release for $12 \mathrm{~h}$. the rheological properties exhibited that all microspheres were free-flowing in nature. SEM photographs confirmed the spherical shape of the microspheres. The results of FTIR, DSC, and XRD revealed that experimental conditions allowed a uniform distribution of the extract within EC microspheres having no significant effect on drug-polymer interaction. The particle size was found to be in the range of 34.25-176.25 $\mu \mathrm{m}$ and showed 
uniform size distribution. Finally, the results of this investigation elucidate that the process and formulation variables could be effectively altered to achieve the desired characteristics of the EC microspheres for novel delivery of the herbal drug.

\section{ACKNOWLEDGMENT}

The author extends her warmest appreciation to her research adviser, Dr. Kalyan Kumar Sen, Principal, Gupta College of Technological Sciences, Asansol, WB, India, for his invaluable support for the completion of this research.

\section{AUTHORS CONTRIBUTIONS}

Ranu Biswas conducted the experiment and prepared the manuscript. Dr. Kalyan Kumar Sen supervised the work and helped in manuscript preparation and correction.

\section{CONFLICT OF INTERESTS}

The authors declare that there are no conflicts of interest

\section{REFERENCES}

1. Parth S, Verma S, Misri P. Global need for novel herbal drug formulations. Int J Pharm Phytopharm Res 2016;8 Suppl 9:1535-44.

2. Kumar A, IIavarasan R, Jayachandran $T$, Deecaraman $M$, Aravindan P, Padmanabhan N, et al. the Anti-diabetic activity of Syzygium cumini and its isolated compound against streptozotocin-induced diabetic rats. J Med Plants Res 2008;2 Suppl 9:246-9.

3. Farswan M, Mazumder PM, Parcha V. Modulatory effect of an isolated compound from Syzygium cumini seeds on biochemical parameters of diabetes in rats. Int J Green Pharm 2014;3:128-33.

4. Banerjee A, Maji B, Mukherjee S, Chaudhuri K, Seal T. In vitro anti-diabetic and antioxidant activities of ethanol extract of Tinospora sinensis. Int J Curr Pharm Res 2017;9 Suppl 2:42-7.

5. Prabakaran C, Shanmugavel G. Antidiabetic activity and phytochemical constituents of syzygium cumini seeds in puducherry region, South India. Int J Pharm Phyto Res 2017;9 Suppl 7:985-9.

6. Mukherjee PK, Harwansh RK, Bhattacharyya S. Bioavailability of herbal products: approach toward improved pharmacokinetics. Evidence-Based Validation Herbal Med 2015;10:217-45.

7. Kumar K, Raj AK. Development and evaluation of floating microspheres of curcumin. Trop J Pharm Res 2012;11 Suppl 5:713-9.

8. Hazra M, Mondal DD, Mondal T, Bhuniya S, Ghosh M. Designing polymeric microparticulate drug delivery system for hydrophobic drug quercetin. Saudi Pharm J 2015;23 Suppl 4:429-36.
9. Akash MSH, Iqbal F, Raza M, Rehman K, Ahmed S, Shahzad Y, et al. Characterization of ethyl cellulose and hydroxypropyl methylcellulose microspheres for controlled release of flurbiprofen. J Pharm Drug Delivery Res 2013;2 Suppl 1:3-10.

10. Guo W, Quan P, Fang L, Cun D, Yang M. Sustained release donepezil loaded PLGA microspheres for injection: preparation, in vitro and in vivo study. Asian J Pharm Sci 2015;10:405-14

11. Nighute AB, Bhise SB. Preparation and evaluation of rifabutin loaded polymeric microspheres. Res J Pharm Technol 2009;2 Suppl 2:371-4.

12. Natarajan V, Natarajan K, Madan B, Sehgal PK. Formulation and evaluation of quercetin polycaprolactone microspheres for the treatment of rheumatoid arthritis. J Pharm Sci 2010;100:195204

13. Subedi G, Shrestha AK, Shakya S. Study of effects of different factors in formulation of micro and nanospheres with solvent evaporation technique. Open Pharm Sci J 2018;5:182-95.

14. Sharma M, Kohli S, Dinda A. In vitro and in vivo evaluation of repaglinide loaded floating microspheres prepared from different viscosity grades of HPMC polymer. Saudi Pharm J 2015;23:675-82.

15. Chitnis KS, Palekar SB, Koppar DR, Mestry DY. Evaluation of Syzygium cumini Linn. Seed formulations available in the market using spectrophotometric and chromatographic techniques. Int J Pharm Sci Res 2012;3 Suppl 2:556-60.

16. Agrawal GR, Wakte P, Shelke S. Formulation, physicochemical characterization and in vitro evaluation of human insulinloaded microspheres as a potential oral carrier. Prog Biomater 2017;6:125-36.

17. Kohli S, Sharma M, Pal A. Ethylcellulose floating microspheres of antidiabetic agent: in vitro and in vivo evaluation. Int J Appl Pharm 2017;9 Suppl 1:44-9.

18. Revathi S, Dhanaraju MD. Fabrication and effect of process variables of sitagliptin microspheres. Asian J Pharm Clin Res 2018;11 Suppl 4:291-7.

19. Navneet $S$, Madan P, Lin S. Effect of process and formulation variables on the preparation of parenteral paclitaxel-loaded biodegradable polymeric nanoparticles: a co-surfactant study. Asian J Pharm Sci 2016;11:404-16.

20. Vyslouzil J, Dolezel P, Kejdusova M, Maskova E, Masek J, Lukac $\mathrm{R}$, et al. Influence of different formulations and process parameters during the preparation of drug-loaded PLGA microspheres evaluated by multivariate data analysis. Acta Pharm 2014;64:403-17.

21. Hoa LTM, Chi NT, Nguyen LH, Chien DM. Preparation and characterization of nanoparticles containing ketoprofen and acrylic polymers prepared by an emulsion solvent evaporation method. J Exp Nano Sci 2011;7 Suppl 2:189-97. 